\title{
Nachhaltigkeitsbewertung für Kleinwasserkraftwerke in China
}

\author{
Felix Tettenborn ${ }^{1} \cdot$ Christian Sartorius $^{1}$
}

Online publiziert: 10. März 2020

○ Der/die Autor(en) 2020

\section{Zusammenfassung}

In China besteht ein hohes hydroenergetisches Potenzial an dezentralen emissionsarmen Lösungen in Form von Kleinwasserkraftwerken. Deren Nutzung kann helfen, soziale Ungleichgewichte abzubauen, gleichzeitig resultieren daraus international kontrovers diskutierte deutliche Eingriffe in Natur und Landschaft und damit in die Gesellschaft. Zur Erfassung und Bewertung der systemischen Auswirkungen der Nutzung von Wasserkraft wurde ein multikriterieller Ansatz erarbeitet, mit dessen Hilfe Planungsprozesse unterstützt und innovative Finanzmittel mobilisiert werden können.

\section{Sustainability Assessment of Hydropower}

\begin{abstract}
In China, there is a large so far untapped hydro energetic potential of decentralized, cluster-controllable and low emission solutions in the form of small hydropower plants. Their use is intended to help to reduce social imbalances. At the same time the utilization of hydropower has a major impact on nature and the landscape and therefore on society. For assessing the systemic impacts of hydropower use in individual sites or hydropower clusters, a multi criteria approach has been compiled. This can better direct planning processes and provide targeted support to administrative approval procedures. The results of the sustainability evaluation can also be a basis to mobilize innovative financial resources.
\end{abstract}

\section{Ausgangslage}

Wasserkraftprojekte umfassen verschiedene Anlagentypen und decken eine Vielzahl von Baugrößen ab. Entsprechend können Art und Ausmaß der Auswirkungen variieren. Die Auswirkungen eines Wasserkraftprojekts stehen in direktem Zusammenhang mit seiner Nachhaltigkeit. Die Aspekte der ökologischen, ökonomischen, sozialen und technischen Nachhaltigkeit sind dabei zum Teil komplementär, zum Teil konkurrierend. Da sich negative Auswirkungen von Wasserkraftprojekten nicht vollständig vermeiden lassen, sind Kompromisse zwischen den verschiedenen Nachhaltigkeitsdimensionen unvermeidlich. Um die Nachhaltigkeit der Wasserkraft erhöhen zu können, müssen daher die Auswirkungen verschiedener Optionen, die bspw. über hydrologische Modellierungen (Merta und Sommer 2019), Standortsoptimierungen (Träbing et al. 2019) oder Opti-

Dieser Beitrag ist ursprünglich erschienen in WasserWirtschaft 109 (2019) [Ausgabe 6].

Felix Tettenborn

felix.tettenborn@isi.fraunhofer.de

1 Fraunhofer-Institut für System- und Innovationsforschung ISI, Breslauer Straße 48, 76139 Karlsruhe, Deutschland mierungen bei der Betriebsführung (Karimanzira und Rauschenbach 2019) erreicht werden können, bewertet werden. Die Ex-ante-Bewertung von Alternativen und die Beurteilung von Zielkonflikten erfolgt als Teil der strategischen Entscheidungsfindung vor der Umsetzung von Projekten. Eine Ex-post-Bewertung kann zur Bestimmung des Nachhaltigkeitsprofils eines bestehenden Projekts bzw. einer Intervention verwendet werden. Bisher gibt es jedoch keinen einheitlichen Ansatz für die Nachhaltigkeitsbewertung von Wasserkraftprojekten. Vielmehr wird eine Vielzahl von Ansätzen und Methoden verwendet, die auf verschiedenen Konzepten und Theorien basiert.

\section{Entwicklung eines methodischen Ansatzes für ein multikriterielles Bewertungskonzept}

\subsection{Vorgehen}

Um sicherzustellen, dass die Entscheidungen zu Umsetzungen von Wasserkraftprojekten und -politiken nachhaltig sind, müssen deren Auswirkungen und Risiken bewertet werden. Die bestehenden Ansätze verwenden verschiedene Methoden und Indikatoren, bewerten unterschiedli- 


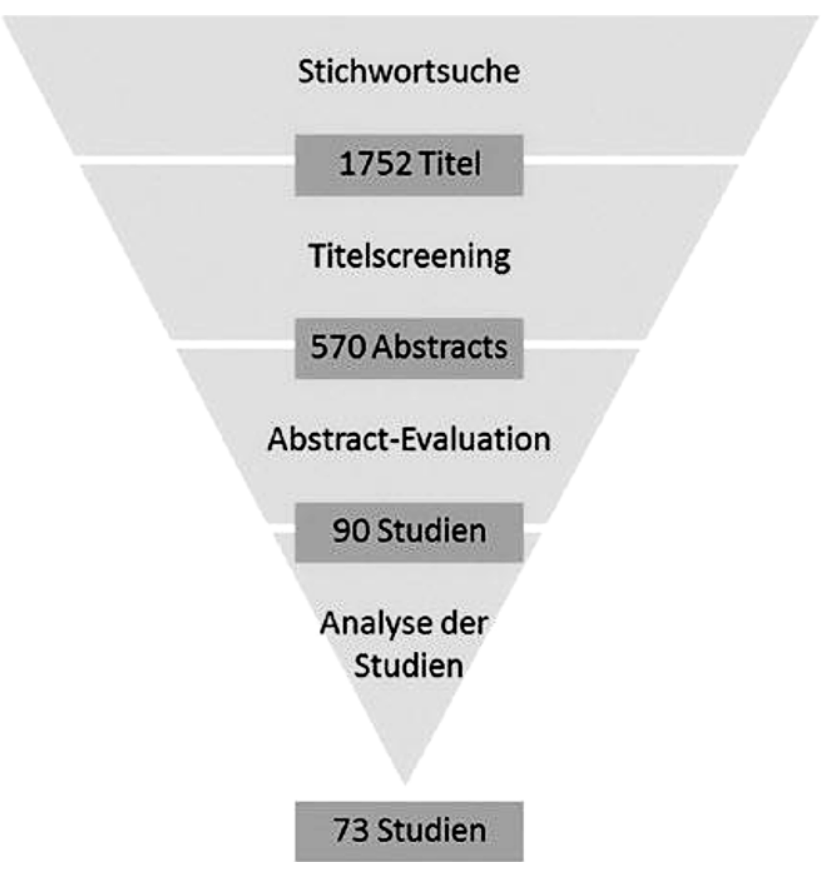

Abb. 1 Schritte des Literaturauswahlverfahrens für die Literaturrecherche

che Technologiearten sowie -größen und sind oft auf eine bestimmte Perspektive beschränkt. Für eine systematische und umfassende Nachhaltigkeitsbewertung müssen jedoch alle relevanten Aspekte berücksichtigt werden. Um diese Aspekte zu bestimmen und bisher verwendete Bewertungsverfahren sowie deren Perspektiven zu identifizieren, wurde innerhalb eines Teilvorhabens des deutsch-chinesischen Forschungsprojektes HAPPI (Rauschenbach 2019) zu Beginn eine Literaturrecherche durchgeführt.

Die in der Literatur dokumentierten Bewertungsverfahren für Wasserkraftanlagen wurden in Kategorien eingeordnet, um eine vergleichende Analyse der verfügbaren Ansätze zu ermöglichen. Dabei wurden die als relevant erachteten Aspekte der Nachhaltigkeit für Wasserkraftprojekte aufgezeigt. Abb. 1 zeigt die Schritte des Literaturauswahlverfahrens.

Aus den vorliegenden Studien wurde ein allgemeiner Bewertungsrahmen mit Bewertungskriterien für die verschiedenen Nachhaltigkeitsdimensionen abgeleitet.

\subsection{Teilergebnis methodischer Ansatz für ein multikriterielles Bewertungskonzept}

Der vorgeschlagene Rahmen für die Nachhaltigkeitsbewertung von Kleinwasserkraftprojekten umfasst vier Hierarchieebenen: die verschiedenen Nachhaltigkeitsdimensionen, die jeweiligen Wirkbereiche, die Kriterien und die spezifischen Indikatoren, mit denen die Kriterien erfasst werden. Von 73 Studien der Literaturübersicht bewerten rund $18 \%$ Kleinwasserkraftprojekte mit einer installier- ten Leistung zwischen 2 und 50MW. Die Ergebnisse der Literaturstudie zeigen, dass die Untersuchungen zu Wasserkraftprojekten verschiedene Anlagentypen berücksichtigten und zusammen die wesentlichen Nachhaltigkeitsaspekte abdecken. Es kristallisierten sich insgesamt 11 Wirkbereiche heraus, die die Nachhaltigkeit von Wasserkraftprojekten beeinflussen. Die in den Bewertungen verwendeten Kriterien wurden anschließend mit diesen Wirkbereichen verknüpft, um rund 30 Kriterienbereiche abzuleiten, die für eine umfassende Nachhaltigkeitsbewertung von Kleinwasserkraft erfasst werden sollten. Diese sind in Abb. 2 aufgeführt und beschreiben den grundsätzlichen Bewertungsraum.

In den verschiedenen bisherigen Bewertungsprojekten werden in der Regel nicht alle Kriterienbereiche gleichermaßen erfasst: So wurden insbesondere die Auswirkungen auf die Arbeitnehmer, d.h. die Gesundheit und Sicherheit der Arbeitnehmer sowie Arbeitnehmerrechte, zumeist nicht berücksichtigt, wobei diese Kriterien im gesellschaftspolitischen Kontext, insb. in Schwellen- und Entwicklungsländern, dazu dienen können auch hierüber Standards für Arbeitsbedingungen und Rechte im Wasserkraftsektor zu adressieren.

Andere Kriterienbereiche, wie spezifische Schadstoffe, lokales Klima, Erschwinglichkeit oder Abfallaufkommen werden nur im Rahmen einzelner Bewertungsstudien erfasst. Einige Kriterien, wie die Fluss-Konnektivität, hydromorphologische Qualität, Beschäftigung und Vertreibung und Umsiedlung sind sowohl im Rahmen von Ex-ante- als auch von Ex-post-Bewertungen relevant. Als Indikatoren kommen bspw. Sedimentation zur Beschreibung der hydromorphologischen Qualität in Frage.

Einige spezifische Auswirkungen werden bislang nicht im Rahmen von Bewertungen für Kleinwasserkraft erfasst: Das Kriterium Infrastruktur wird bei den Bewertungen von Kleinwasserkraftprojekten bislang vor allem in Bezug auf die Wasserversorgungsstruktur verstanden, während die Auswirkungen auf die öffentliche Infrastruktur, d.h. Straßen und öffentliche Gebäude, nicht berücksichtigt werden. Die Infrastruktur könnte aber bspw. vorab über die öffentliche Infrastruktur im Bereich des Anlagenbaus und im Nachhinein durch Veränderungen der öffentlichen Infrastruktur bewertet werden. Für eine Weiterentwicklung des Rahmens für die Nachhaltigkeitsbewertung sollten daher angepasste Ex-ante- und Ex-post-Indikatoren formuliert werden, die die jeweiligen Kriterien abdecken.

Ein Vergleich der von den Wasserkraftbewertungen verwendeten Indikatoren und der betrachteten Auswirkungen ergab, dass sich die Auswirkungen von Kleinwasserkraftprojekten nicht unbedingt von denen anderer Wasserkraftanlagengrößen in der Art der Auswirkungen unterscheiden, sondern lediglich in ihrer Größenordnung.

Eine umfassende Beschreibung der Kriterien sowie eine Vorgehensempfehlung zur Durchführung einer Nachhaltig- 

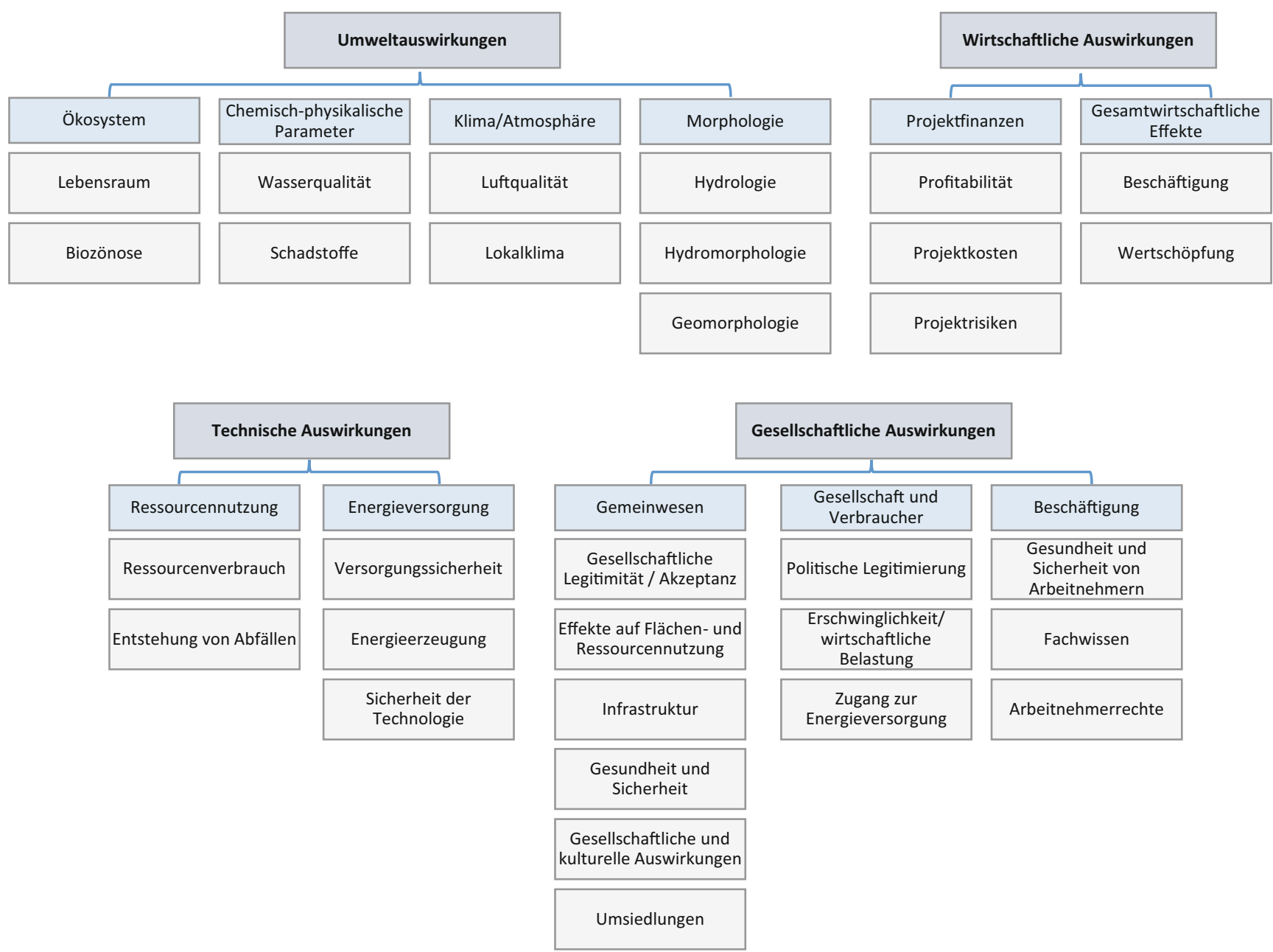

Abb. 2 Rahmen für die Nachhaltigkeitsbewertung von Kleinwasserkraftprojekten; die Ebenen sind in absteigender Reihenfolge angeordnet: die Nachhaltigkeitsdimensionen, die Wirkbereiche und die Kriterienbereiche

keitsbewertung erfolgten im Rahmen von (Tettenborn 2018) und der darin enthaltenen praxisorientierten Leitfäden.

\section{Erhebung sozioökonomischer Nachhaltigkeitsaspekte}

\subsection{Vorgehen}

Zur exemplarischen Erfassung sozioökonomischer Effekte aufgrund von Kleinwasserkraftprojektumsetzungen im Rahmen des SHSF-Programms der chinesischen Regierung (SHSF: small hydropower substituting fuel) wurde in Abstimmung mit chinesischen Projektpartnern ein Fragebogen erstellt. Abb. 3 zeigt die Kriterien der sozialen Nachhaltigkeit des entwickelten Bewertungsrahmens auf. Die farbigen Blöcke zeigen an, welche Aspekte über den Fragebogen abgedeckt wurden.

Aspekte von Arbeitnehmergesundheit, Arbeitssicherheit und Arbeitsrechte waren in diesem Fragebogen, der auf die breite Öffentlichkeit abzielte, nicht enthalten. Ebenso gab es, entsprechend Abstimmung mit den chinesischen Partnern, keine Fragen, die auf Umsiedlungsaspekte oder auf die politische Legitimität ausgerichtet waren. Letztere kann im Zusammenhang mit der SHSF-Förderung als gegeben angesehen werden.

Fragen zur Erfassung der ökonomischen Nachhaltigkeit der Kleinwasserkraft betreffen überwiegend Effekte von Wertschöpfungsketten. Die verbleibenden Fragen befassen sich mit Beschäftigungsmöglichkeiten und dem Beitrag von Anwohnern zum Bau von Kleinwasserkraftanlagen. Projektökonomische Aspekte wurden nicht berücksichtigt, da diese bereits im Rahmen der Projektplanung ex-ante berücksichtigt wurden. Abb. 4 gibt einen Überblick über die im Fragebogen abgedeckten ökonomischen Nachhaltigkeitskriterien.

An folgenden Standorten wurden von den chinesischen Partnern Daten erhoben: 


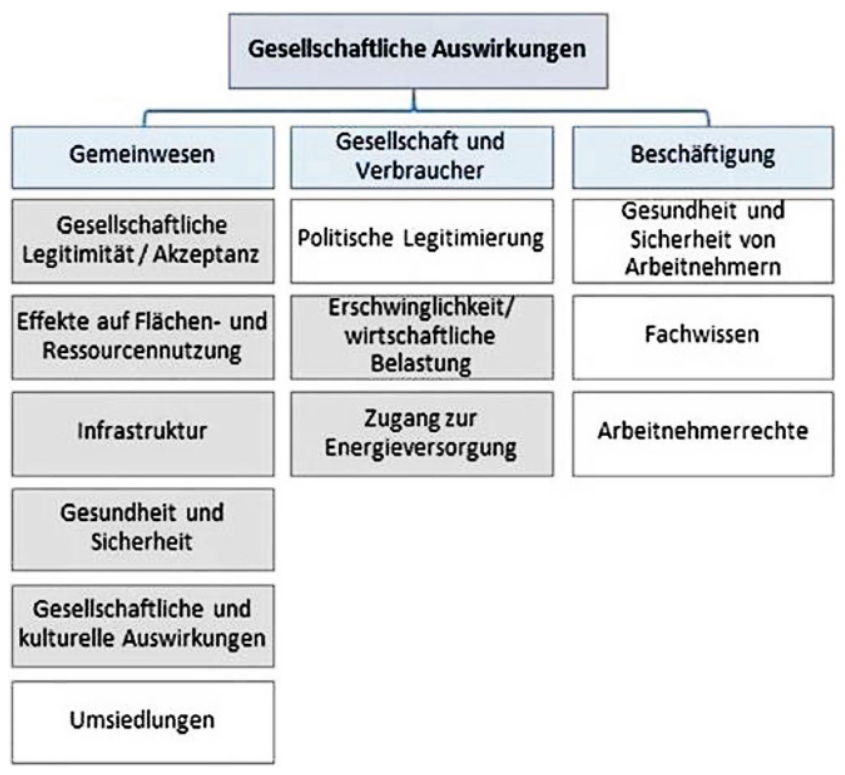

Abb. 3 Soziale Nachhaltigkeitskriterien des SHSF-Fragebogens

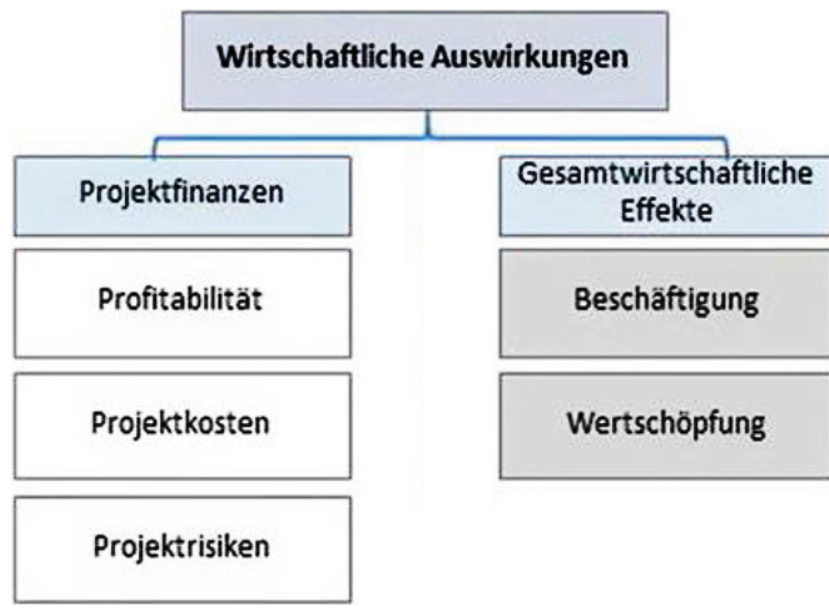

Abb. 4 Wirtschaftliche Nachhaltigkeitskriterien des SHSF-Fragebogens

- Fujiang, Kleinwasserkraftanlage mit Förderung durch das SHSF Programm;

- Wengbao, in der Nähe einer Kleinwasserkraftanlage ohne Förderung durch das SHSF Programm und

- Dexing, ohne Anbindung an eine Kleinwasserkraftanlage.

Von den Antworten aus insgesamt 300 Haushalten konnten laut Aussage der chinesischen Partner 235 Antwortsets ausgewertet werden. Dies entspricht einer Verwertungsquote von rund $80 \%$. Das Fraunhofer ISI war an der Befragungsdurchführung nicht beteiligt, die Ergebnisse wurden von den Projektpartnern in zusammengefasster Form übermittelt.

\subsection{Teilergebnis Erhebung sozioökonomischer Nachhaltigkeitsaspekte}

Laut der Erhebung der Beijing Forestry University (BFU) ist das Gesamteinkommen der privaten Haushalte in Fujiang höher als in Haushalten mit Anbindung an ein Wasserkraftwerk außerhalb des SHSF-Programms (Wengbao) bzw. in Haushalten ohne Anbindung an ein Wasserkraftwerk (Dexing). Die Ergebnisse der BFU zeigen weiter, dass die Gesamtzufriedenheit mit dem SHSF-Programm groß ist. Auf die Frage, ob und wie sich die Lebensqualität nach Anbindung der Kleinwasserkraftanlage verändert hat, gab die Mehrheit in Fujiang (77\%) an, dass ihr Leben jetzt bequemer sei; $23 \%$ gaben an, keine signifikante Veränderung wahrzunehmen. In den Vergleichsregionen Wengbao und Dexing fiel die Zustimmung zu einer erhöhten Lebensqualität mit $40 \%$ bzw. $27 \%$ geringer aus, während die Angaben zur Wahrnehmung von „keinen signifikanten Veränderungen“" bei $60 \%$ bzw. $73 \%$ lagen.

Die Akzeptanz des SHSF-Programms ist entsprechend BFU-Erhebung ebenfalls groß. Das SHSF-Programm wird offensichtlich als eine Gelegenheit angesehen, erschwingliche und saubere Energie zu erhalten. Seitens Zhang et al. wird herausgestellt, dass fortlaufende Bildungsprogramme und gute Kommunikation seitens Politik erforderlich sind, um den Programmerfolg zu erhalten (Zhang et al. 2017).

\section{Abgleich mit nationalen und globalen Nachhaltigkeitszielen}

\subsection{Vorgehen}

Ergänzend zu den Arbeiten zu der exemplarischen Bewertung sozioökonomischer Effekte von Kleinwasserkraftanlagen wurde in Anlehnung an (Sartorius et al. 2019) ein Abgleich der Ziele der Vereinten Nationen (UN) für nachhaltige Entwicklung (Sustainable Development Goals; SDG) mit der chinesischen Nachhaltigkeitsstrategie und landesspezifischen Nachhaltigkeitsindikatoren sowie mit den Zielen des SHSF-Programms durchgeführt.

Die insgesamt 17 Ziele für nachhaltige Entwicklung der UN sind in der sogenannten Agenda 2030 festgehalten (UN 2015b), Transformation unserer Welt: die Agenda 2030 für nachhaltige Entwicklung. Resolution der Generalversammlung,verabschiedet am 25. September 2015). Es handelt sich dabei um politische Zielsetzungen, die der Sicherung einer nachhaltigen Entwicklung auf ökonomischer, sozialer sowie ökologischer Ebene dienen sollen. Die Ziele wurden in Anlehnung an den Entwicklungsprozess der Millennium Development Goals (MDG) entworfen, bauen auf diesen auf und traten Anfang 2016 mit einer Laufzeit von 15 Jahren (bis 2030) in Kraft. Im Unterschied zu den MDG, 
die insbesondere auf Veränderungen in Entwicklungsländern abzielten, gelten die SDG für alle Staaten. Die SDG wurden in einem breiten, zweijährigen Konsultationsprozess entwickelt, bei dem nicht nur die Politik, sondern auch die Wissenschaft, Zivilgesellschaft und Privatwirtschaft aller UN-Länder die Möglichkeit hatten, sich einzubringen. Sie werden bspw. laut Bundesministerium für wirtschaftliche Zusammenarbeit und Entwicklung als Meilenstein in der jüngeren Geschichte der Vereinten Nationen bezeichnet (BMZ 2019).

Zur Konkretisierung der 17 Ziele wurde ein Katalog von 169 Zielvorgaben verabschiedet, darunter u. a. das Auslaufen der Subventionen für fossile Energien (UN, Resolution der Generalversammlung, 69/315. 2015a). Die Umsetzung der Agenda 2030 liegt in der Verantwortung der Länder. Mittels eines Überprüfungsmechanismus unter dem Dach des High Level Political Forums on Sustainable Development der UN (HLPF) (BMZ 2019) sollen die Fortschritte anhand von Indikatoren nachverfolgt werden.

In China wurde 1994 im Zusammenhang mit der World Commission on Environment and Development (UN WCED) in Rio, Brasilien, der erste Meilenstein zur nachhaltigen Entwicklung mit der Veröffentlichung der ersten nationalen Nachhaltigkeits-Strategie „Chinas Agenda 21 - White Paper on China's Population, Environment and Development in the 21st Century" gelegt. Das Strategiepapier hält fest, dass Bevölkerung, Ressourcen und Umwelt drei Eckpfeiler sind, die die Volkswirtschaft, die soziale Sicherheit und den Lebensstandard stützen. Angesichts der großen Bevölkerung, der Ressourcenknappheit und dem fragilen ökologischen Umfeld werden Wissenschaft und Technologie eine entscheidende Rolle bei der Umsetzung der Strategie für nachhaltige Entwicklung und bei der Integration in die wirtschaftliche Entwicklung zugeschrieben.

$\mathrm{Zu}$ den Zielen der chinesischen Agenda 21 gehören die Verbesserung der Infrastruktur, die umfassende Förderung der sozialen Entwicklung, die Schaffung nationaler politischer und rechtlicher Systeme für nachhaltige Entwicklung sowie die Verbesserung der Umwelt. Auf diese Weise wird ein Gleichgewicht zwischen der wirtschaftlichen, sozialen und ökologischen Entwicklung des Landes angestrebt.

Eine stufenweise Konkretisierung der chinesischen Agenda 21 erfolgte auf nationaler Ebene vor allem über die regelmäßig erstellten Fünfjahrespläne (FYP), in denen die landesweiten Entwicklungsinitiativen von Wirtschaft und Gesellschaft definiert werden, und über die nationalen Maßnahmen und die Entwicklung der chinesischen Politik vorangetrieben werden. Auch wenn die Veröffentlichung der chinesischen Agenda 21 im Laufe des 8. FYP (1991-1995) erfolgte, thematisierte erstmalig der 10. FYP (2000-2005) das Thema Umweltschutz im Rahmen der nationalen Zielsetzung als eines der Hauptziele. Ein weiterer Schritt der chinesischen Regierung zum Aufbau einer nationalen Strategie für nachhaltige Entwicklung erfolgte mit der Initiierung des ,Aktionsprogramms für nachhaltige Entwicklung in China im frühen 21. Jahrhundert“". Das Programm wurde in der späteren Phase des 10. FYP veröffentlicht. Es spiegelt daher dessen Ziele wider und beeinflusste die Erstellung des 11. (2006-2010) und 12. FYP (2011-2015). Mit den allgemeinen Zielen und Leitlinien des Programms wurde nicht nur weiterhin das Ziel verfolgt, das Gleichgewicht zwischen sozioökonomischer Entwicklung, Bevölkerung, Ressourcen und Umwelt zu fördern, sondern auch der Aufbau von Kapazitäten für nachhaltige Entwicklung als eines der obersten Ziele genannt.

\subsection{Teilergebnis Aggregierte Betrachtung der Ziele von Nachhaltigkeitsstrategien}

Von den 169 SDG-Zielvorgaben lassen sich bei 63 Unterzielen Verbindungen mit den Zielen des SHSF-Programms und mit den Zielen der chinesischen Nachhaltigkeitsstrategie knüpfen. Zum Teil sind diese sehr genau passend, bspw. bei den Unterzielen von SDG 7 ,Nachhaltige und moderne Energie für alle - Zugang zu bezahlbarer, verlässlicher, nachhaltiger und zeitgemäßer Energie für alle sichern“.

Grundsätzlich und auf qualitativer Ebene zeigt sich, dass sich über die Ziele des SHSF-Programms Verbesserungen bzgl. der SDG ergeben, die bspw. über den erarbeiteten multikriteriellen Bewertungsansatz erfasst werden können. Absolute Aussagen hinsichtlich des Zielerreichungsgrades sind aus verschiedenen Gründen aber nicht möglich:

So beziehen sich viele der SDG auf die Gesamtbevölkerung. Das SHSF-Programm hingegen wurde zu dem Zweck aufgesetzt, die Energieversorgung in bestimmten Gebieten des zu entwickelnden ländlichen Raums unter den dort herrschenden spezifischen Randbedingungen zu verbessern. Dabei ist es grundsätzlich zwar möglich, die Systemgrenzen der Bewertung zur Erfassung der Zielerreichung so zu erweitern, dass eine Aussage bzgl. einer Region oder eines Land getroffen werden kann. Voraussetzung für diese Erweiterung ist aber u.a., dass für das Bewertungsgebiet ein einheitlicher Rahmen gilt, da die Teilnutzen auf der Grundlage gesetzlich oder politisch festgelegter Grenzbzw. Zielwerte erreicht werden. Gleichzeitig bedeutet die Ausweitung der Systemgrenzen, dass der Gesamtzustand des vorhandenen Systems anhand von öffentlich verfügbaren Statistiken beurteilt werden muss, die i.d.R. Durchschnittswerte darstellen. Besondere Umstände, die für die Errichtung einer Anlage an einem bestimmten Ort sprechen, können nicht berücksichtigt werden und die Vorteile der Alternative kämen dadurch in vielen Fällen nicht zum Tragen.

In anderen Fällen ist die durchschnittliche Ausprägung eines Indikators innerhalb eines Referenzgebietes Grundlage für die Bestimmung der Teilnutzwerte. Da in diesem 
Fall zusätzlich der gesetzliche Rahmen als ,gemeinsamer Faktor" eines Untersuchungsgebietes wegfällt, trifft das Argument, dass lokale Besonderheiten durch die Ausweitung der Systemgrenzen und die Verwendung von Durchschnittswerten für die Indikatoren nivelliert werden, umso mehr zu. Selbst wenn die erforderlichen Daten verfügbar wären, wäre eine Bewertung auf dieser aggregierten Ebene somit nicht sinnvoll.

Durch den entwickelten Bewertungsansatz ist außerdem auch nur deshalb eine relative Gegenüberstellung von Veränderungen möglich, weil die Energieinfrastrukturen zu allen SDG außer SDG 7 nur Teilbeiträge zur Zielerreichung leisten können. Bei einem Abgleich mit den Kriterien, die bei der vergleichenden Bewertung von alternativen Energieinfrastrukturmaßnahmen mit dem entwickelten Bewertungsansatz zur Anwendung kommen, zeigt sich, dass neben den SDG 7.1, 7.2 und 7.a vor allem SDG 9.1 „Aufbau einer hochwertigen, verlässlichen, nachhaltigen, widerstandsfähigen und für alle erschwinglichen Infrastruktur" betroffen ist. Die Energieinfrastruktur ist in diesem Zusammenhang allerdings nur ein Teil der gesamten Infrastruktur, es werden aber auch übergeordnete Aspekte, wie Verlässlichkeit und Widerstandsfähigkeit, angesprochen, die nur eingeschränkt für einen Infrastrukturbereich alleine behandelt werden können. Daneben kommen in den weiteren SDG Wirkungen der Energieinfrastruktur auf andere Schutzgüter zum Tragen, die eigenständige Ziele darstellen. Im Falle der SDG 3.2, 3.3 und 3.9 ist das Schutzgut Gesundheit betroffen, bei den SDG 2.4, 7.3 und 8.4 die Schonung der natürlichen Ressourcen einschließlich der Rohstoffbasis und bei SDG 7.2 sowie 13.2 der Klimaschutz.

Durch die Anwendung des entwickelten Bewertungsansatzes kann eine Aussage darüber getroffen werden, ob und zu welchen SDG die Veränderung der Energieinfrastruktur bspw. durch die Einführung einer innovativen und nachhaltigen Energiegewinnung einen Beitrag leistet und ob dieser Beitrag jeweils positiv oder negativ ist. Auf die Frage, ob die Einführung einer alternativen Energieinfrastruktur insgesamt zu einer nachhaltigen Entwicklung führt, liefert dieses Vorgehen nur dann eine eindeutige Antwort, wenn alle relevanten SDG entweder auf eine Verbesserung oder eine Verschlechterung hindeuten. Weisen die Veränderungen der SDG hingegen in verschiedene Richtungen, dann ist eine Aggregierung über alle Ziele hinweg nicht möglich, weil weder die Möglichkeit einer Normierung der verschiedenen Ziele besteht, noch eine Gewichtung vorgesehen ist. Selbst die einfache Saldierung von Zielen mit positiver Veränderung mit solchen mit negativer Veränderung ist unter diesen Bedingungen nicht zulässig, da sie implizit eine Gleichgewichtung unterstellen würde.

Für einen Abgleich nachhaltigkeitsrelevanter Aspekte und Erfolge in Bezug auf die nationalen Zielsetzungen konnten die Ziele des 10. und des 12. FYP verwendet wer- den. Die nachgewiesenen Erfolge stammen hauptsächlich aus (Kong et al. 2015) sowie der vom Fraunhofer ISI erarbeiteten und von der BFU durchgeführten Erhebung (Tettenborn 2018). Aus übersetzten Projektdokumenten wurden weitere Nachhaltigkeitsaspekte aufgenommen. Auch hier zeigt sich grundsätzlich und auf qualitativer Ebene, dass sich über die Beiträge des SHSF-Programms Verbesserungen bzgl. der nationalen Zielvorgaben ergeben, die über den erarbeiteten multikriteriellen Bewertungsansatz erfasst werden können. Offensichtlich ist, dass in dem 12. FYP erheblich mehr Zielvorgaben enthalten sind, bei denen das SHSF-Programm im Rahmen des entwickelten Bewertungsansatzes einen positiven Beitrag leisten kann, als noch im 10. FYP. Da über den Bewertungsansatz umfassende Kriterien zur Bewertung der Nachhaltigkeit erfasst sind, zeigt sich, dass der Stellenwert von Nachhaltigkeitsaspekten zwischen den beiden Fünfjahresplänen weiter stark angestiegen ist und dass sich die chinesische Regierung intensiv bemüht hat, bestehende Lücken und Herausforderungen hinsichtlich einer nachhaltigen Entwicklung zu analysieren, um Chinas Strategien für die Zukunft zu verbessern und anzupassen. Absolute Aussagen hinsichtlich des Zielerreichungsgrades sind wie oben dargestellt aber ebenfalls nicht möglich.

\section{Zusammenfassung}

In Abhängigkeit vom Begriffsverständnis zur Nachhaltigkeit kann die Einordnung von Wasserkraftprojekten unter Nachhaltigkeitsaspekten variieren. Für eine systematische Entwicklung eines Nachhaltigkeitsbewertungsansatzes muss daher festgelegt werden, wie die Dimensionen und Prinzipien der Nachhaltigkeit im Rahmen eines Vorhabens verstanden werden. Größe und Anlagentyp sowie Perspektive der Entscheidungsfindung haben darüber hinaus zusätzlich Auswirkungen auf die Durchführung einer Nachhaltigkeitsbewertung. Daher wurde im Rahmen des HAPPIProjektes ein umfassender multikriterieller Ansatz zur Erfassung, Messung und Bewertung der systemischen Auswirkungen der Nutzung von Wasserkraft in Bezug auf einzelne Standorte bzw. auf Wasserkraft-Cluster erarbeitet. Exemplarisch wurde eine Bewertung von sozioökonomischen Auswirkungen von Kleinwasserkraftwerken und des SHSFProgramms am Pilotstandort Fujiang in Majiang County in der Provinz Guizhou durchgeführt.

Zusätzlich wurden die für eine Nachhaltigkeitsbewertung relevanten nationalen Rahmenbedingungen (u. a. nationale Nachhaltigkeitsstrategie; Projektentwicklungszyklus für Kleinwasserkraftanlagen; relevante Akteure und deren Interessen sowie Einflussmöglichkeiten) untersucht. Die Kriterien der Nachhaltigkeitsbewertung wurde mit nationalen und globalen Nachhaltigkeitszielen abgeglichen, 
wie den Nachhaltigkeitszielen der Vereinten Nationen (SDG), die die Politik und Umsetzungen in nachhaltigkeitsrelevanten Bereichen von Gesellschaft und Umwelt in den nächsten fünfzehn Jahren beeinflussen und stimulieren werden. Für viele Ziele, die über den erarbeiteten multikriteriellen Bewertungsansatz erfasst werden können, gibt es unmittelbare, zum Teil auch indirektere Verbindungen, bei denen das SHSF-Programm und Kleinwasserkraftprojekte zur Erreichung der globalen und der nationalen Nachhaltigkeitsziele von China beitragen können. Absolute Aussagen hinsichtlich eines möglichen Zielerreichungsgrad sind jedoch aus verschiedenen, oben genannten Gründen nicht möglich.

Das Verständnis und die Bewertung dieser Aspekte dient u. a. dazu, die nationalen Planungsprozesse zu fördern, behördliche Genehmigungsverfahren hinsichtlich einer verstärkten Beachtung von Nachhaltigkeitszielen zu unterstützen und die Mobilisierung innovativer Finanzmittel zu ermöglichen.

Die Internet-Präsenz auf www.happishp.com bietet langfristigen Zugriff auf die im Projekt erarbeiteten Leitlinien.

Danksagung Der erfolgreiche Abschluss des Vorhabens ist dem chinesischen und deutschen Projektteam und der Projektleitung zu verdanken.

Förderung Das diesem Bericht zugrundeliegende Vorhaben wurde vom Bundesministerium für Bildung und Forschung (BMBF) im Rahmen der FONA-Fördermaßnahme CLIENT, Internationale Partnerschaften für nachhaltige Klimaschutz- und Umwelttechnologien und -dienstleistungen, unter dem Förderkennzeichen 02WCL1307 gefördert.

Funding Open Access funding provided by Projekt DEAL.

Open Access Dieser Artikel wird unter der Creative Commons Namensnennung 4.0 International Lizenz veröffentlicht, welche die Nutzung, Vervielfältigung, Bearbeitung, Verbreitung und Wiedergabe in jeglichem Medium und Format erlaubt, sofern Sie den/die ursprünglichen Autor(en) und die Quelle ordnungsgemäß nennen, einen Link zur Creative Commons Lizenz beifügen und angeben, ob Änderungen vorgenommen wurden.

Die in diesem Artikel enthaltenen Bilder und sonstiges Drittmaterial unterliegen ebenfalls der genannten Creative Commons Lizenz, sofern sich aus der Abbildungslegende nichts anderes ergibt. Sofern das betreffende Material nicht unter der genannten Creative Commons Lizenz steht und die betreffende Handlung nicht nach gesetzlichen Vorschriften erlaubt ist, ist für die oben aufgeführten Weiterverwendungen des Materials die Einwilligung des jeweiligen Rechteinhabers einzuholen.

Weitere Details zur Lizenz entnehmen Sie bitte der Lizenzinformation auf http://creativecommons.org/licenses/by/4.0/deed.de.

\section{Literatur}

BMZ, Bundesministerium für wirtschaftliche Zusammenarbeit und Entwicklung (Hrsg) (2019) Internationale Ziele - Die Agenda 2030 für nachhaltige. www.bmz.de/de/ministerium/ziele/2030_ agenda/index.html. Zugegriffen: 30. Nov. 2018

Karimanzira D, Rauschenbach T (2019) Optimale Betriebsführung von. Wasserwirtschaft 109(6):43-50

Kong Y, Wang J, Kong Z et al (2015) Small hydropower in China: the survey and sustainable future. Renew Sustain Energy Rev 48:425-433

Merta M, Sommer H (2019) Hydrologische Modellierung für die Optimierung von Kleinwasserkraftwerkskaskaden. Wasserwirtschaft 109(6):22-28

Rauschenbach T (2019) Bewertung und Verbesserung von Kleinwasserkraftwerken in China. Wasserwirtschaft 109(6):12-15

Sartorius C, Hillenbrand T, Niederste-Hollenberg J (2019) Multikriterielle Bewertung von Wasserinfrastruktursystemen im Kontext der SDGs. In: Leal W (Hrsg) Handbuch der Nachhaltigkeitsziele: Mensch, Planet, Wohlstand, Frieden und Partnerschaft. Springer, Heidelberg

Tettenborn F (2018) Nachhaltigkeitsbewertung/Klima und Sozioökonomie. In: Rauschenbach T (Hrsg) Schlussbericht zum FuE-Verbundvorhaben HAPPI (Förderkennzeichen 02WCL1307). Fraunhofer IOSB, Ilmenau

Träbing K, Dickel S, Theobald S (2019) Bauwerkshydraulik und Standortoptimierung für Kleinwasserkraftwerke in China. Wasserwirtschaft 109(6):36-42

UN (Hrsg) (2015a) Resolution der Generalversammlung, 69/315. Entwurf des Ergebnisdokuments des Gipfeltreffens der UN zur Verabschiedung der Post-2015-Entwicklungsagenda, Zielvorgabe 12

UN (Hrsg) (2015b) Transformation unserer Welt: die Agenda 2030 für nachhaltige Entwicklung. Resolution der Generalversammlung,verabschiedet am 25. September 2015. www.un.org/Depts/ german/gv-70/band1/ar70001.pdf. Zugegriffen: 3. Feb. 2020

Zhang X, Zha T, Zhao Y et al (2017) Sustainable effects of small hydropower substituting firewood program in Majiang County, Guizhou Province, China. Sustainability 9(6):Art. 988 